\title{
Nitrofurantoin-induced pulmonary fibrosis
}

\author{
John O’Bryen, Gwynne Hannay, \\ Sarah Gleeson

\section{CASE}

A woman aged 75 years presented to a general practitioner (GP) with a dry cough and increasing exertional dyspnoea for the preceding three years. She was now short of breath walking 50 metres. Her cough was largely unproductive but could produce a small amount of clear sputum in the morning. She had experienced episodes of atypical chest pain. There was no history of haemoptysis, orthopnoea, paroxysmal nocturnal dyspnoea or pedal oedema. She had never smoked, received chemotherapy or had occupational dust exposures or recurrent chest infections. Her medical history included use of nitrofurantoin $50 \mathrm{mg}$ daily for the preceding six years as prophylaxis for urinary tract infections.

On examination, she had a blood pressure of 120/80 $\mathrm{mmHg}$ and oxygen saturation of $95 \%$ on room air. She did not have digital clubbing, and heart sounds were dual with no murmurs. There were fine inspiratory crackles throughout the lung fields, particularly at the bases, and reduced breath sounds. The patient was referred for computed tomography (CT) of the chest, which showed advanced pulmonary fibrosis (Figure 1).

\section{QUESTION 1}

What are the causes of pulmonary fibrosis?

\section{QUESTION 2}

What medications are known to cause pulmonary fibrosis?

\section{ANSWER 1}

Causes of pulmonary fibrosis include idiopathic pulmonary fibrosis, autoimmune, environmental (hypersensitivity pneumonitis), occupational (pneumoconiosis), drug-induced pulmonary fibrosis (DIPF) and radiation. Given this patient's history, the lead differentials are idiopathic pulmonary fibrosis and DIPF.

\section{ANSWER 2}

Many offending medications are cancer therapies, rheumatological therapies and antibiotics. ${ }^{1}$ Those commonly seen in general practice include amiodarone, nitrofurantoin and methotrexate. When assessing for DIPF, it is important to obtain a detailed past and present medication list, including chemotherapy, non-prescription medications and recreational drugs. Resources such as the Pneumotox application (https://pneumotox.com) are helpful in identifying culprit medications. ${ }^{2}$

\section{CASE CONTINUED}

Given the possibility of DIPF, the nitrofurantoin was ceased, and the patient felt an improvement of her symptoms over the following weeks. She was referred to a respiratory physician and cardiologist. The cardiologist arranged a myocardial perfusion scan and echocardiography, which helped exclude ischaemic heart disease and heart failure. The echocardiogram did not show pulmonary hypertension. The respiratory physician arranged complex lung function tests, which showed a
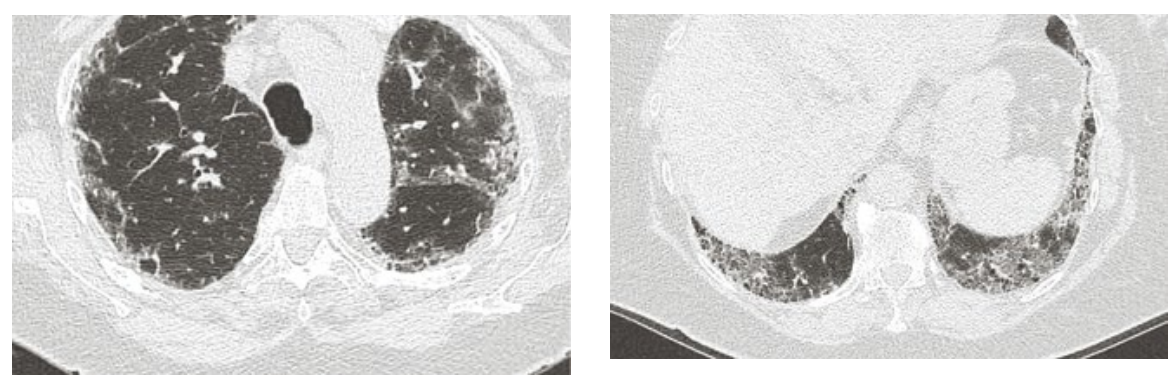

Figure 1. Computed tomography images of the lungs demonstrating peripheral reticular opacification, which involves the upper (image on left) and lower lobes (image on right) with a slight basal predominance. There is honeycombing present and minimal ground glass changes. 
mild restrictive ventilatory deficit with a moderate impairment in gas transfer (after correction for volume restriction). The results are shown in Figure 2.

A pulmonary fibrosis association screening panel was negative. Repeat CT of the chest three months later showed no radiological progression of the pulmonary interstitial fibrosis. At this time, the patient reported her shortness of breath on exertion had improved to
300 metres. She had received vaccination for influenza and pneumococcus. She was encouraged to remain a non-smoker and to engage in pulmonary rehabilitation. Given the improvement in her symptoms following the cessation of nitrofurantoin, a diagnosis of DIPF was made. The medication adverse event was reported to the Therapeutic Goods Administration (TGA).

\section{QUESTION 3}

What screening blood tests could be used to investigate causes of pulmonary fibrosis?

\section{QUESTION 4}

What monitoring is required for patients receiving a medication known to cause DIPF?

QUESTION 5

Which medication adverse events should be reported to the TGA?

\section{Flow Volume Loops}

\begin{tabular}{|c|c|c|c|c|c|c|c|c|c|c|c|c|c|}
\hline \multirow{2}{*}{ Measurement date } & & \multirow[t]{2}{*}{ Predicted } & \multirow[t]{2}{*}{ Pred LL } & \multirow[t]{2}{*}{ Pred UL } & \multirow{2}{*}{$\begin{array}{c}\text { Pre } \\
14.05 .19\end{array}$} & \multirow[t]{2}{*}{$\%$ Pred } & \multirow{2}{*}{$\begin{array}{c}\text { Post } \\
14.05 .19\end{array}$} & \multirow[t]{2}{*}{ \%Pred } & \multirow[t]{2}{*}{$\%$ Chg } & \multicolumn{3}{|c|}{$-3-2 \mathbf{Z}_{7}$ Score $_{2}$} & \multirow[t]{2}{*}{ Z-Score } \\
\hline & & & & & & & & & & & & & \\
\hline FEV 1 & $\mathbf{L}$ & 1.76 & 1.25 & 2.24 & 1.42 & 80.9 & 1.43 & 81.7 & 1.0 & - & & & -1.11 \\
\hline FVC & $\mathbf{L}$ & 2.27 & 1.62 & 2.95 & 1.71 & 75.3 & 1.68 & 73.9 & -1.9 & - & & & -1.41 \\
\hline FEV $1 \%$ FVC & $\%$ & 78.11 & 63.98 & 90.32 & 83.19 & 106.5 & 85.63 & 109.6 & 2.9 & & - & 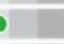 & 0.66 \\
\hline PEF & $\mathrm{L} / \mathrm{s}$ & 4.95 & 3.46 & 6.43 & 6.49 & 131.1 & 6.13 & 124.0 & -5.5 & & & 0 & 1.71 \\
\hline MEF 50 & L/s & 2.98 & 1.17 & 4.79 & 3.06 & 102.6 & 3.61 & 121.0 & 17.9 & & - & & 0.07 \\
\hline MFEF $75 / 25$ & $\mathrm{~L} / \mathrm{s}$ & 1.52 & 0.67 & 2.78 & 1.71 & 112.1 & 2.09 & 137.5 & 22.7 & & - & & 0.28 \\
\hline PIF & $\mathrm{L} / \mathrm{s}$ & & & & 2.76 & & 2.52 & & -8.9 & & & & \\
\hline FIF 50 & L/s & & & & 1.50 & & 2.07 & & 38.2 & & & & \\
\hline \multicolumn{14}{|l|}{ Lung Volumes } \\
\hline TLC & $\mathbf{L}$ & 4.18 & 3.19 & 5.16 & 2.63 & 63.1 & & & & - & & & -2.57 \\
\hline FRCpleth & L & 2.46 & 1.63 & 3.28 & 1.33 & 54.2 & & & & 0 & & & -2.25 \\
\hline RV & $\mathbf{L}$ & 1.93 & 1.36 & 2.51 & 0.86 & 44.6 & & & & D & & & -3.06 \\
\hline RV \% TLC & $\%$ & 44.46 & 34.87 & 54.05 & 32.76 & 73.7 & & & & 0 & & & -2.01 \\
\hline VC & $\mathbf{L}$ & 1.96 & 1.27 & 2.65 & 1.77 & 90.5 & & & & & - & & -0.44 \\
\hline ERV & $\mathbf{L}$ & 0.52 & 0.52 & 0.52 & 0.47 & 89.3 & & & & & & & \\
\hline \multicolumn{14}{|l|}{ Transfer Factor } \\
\hline DLCO_SB & & 16.27 & 12.17 & 22.29 & & & 6.85 & 42.1 & & D & & & -5.30 \\
\hline DLCOCSB & & 16.27 & 12.17 & 22.29 & & & 6.85 & 42.1 & & $D$ & & & -5.30 \\
\hline KCO_SB & & 4.28 & 3.25 & 5.47 & & & 2.88 & 67.3 & & 0 & & & -2.31 \\
\hline KCOc_SB & & 4.28 & 3.25 & 5.47 & & & 2.88 & 67.3 & & 0 & & & -2.31 \\
\hline VA_SB & & 3.83 & 3.04 & 4.80 & & & 2.38 & 62.2 & & D & & & -3.32 \\
\hline $\mathrm{Hb}$ & & & & & & & 13.40 & & & & & & \\
\hline
\end{tabular}

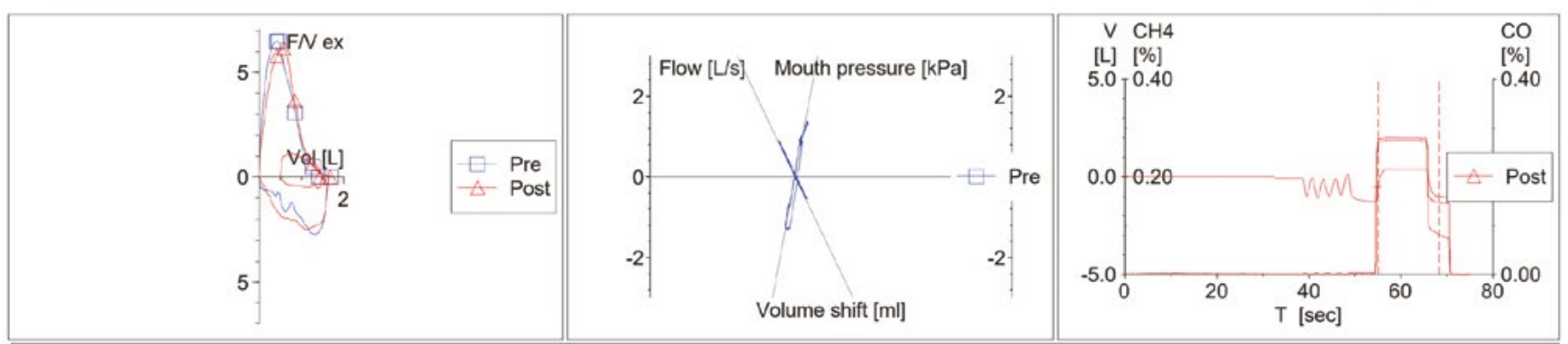

\section{Figure 2. Complex lung function test results}

$\mathrm{CH}$, methane; Chg, change; $C O$, carbon monoxide; DLCO_SB, single-breath diffusing lung capacity for carbon monoxide; DLCOcSB, single-breath diffusing lung capacity for carbon monoxide corrected for haemoglobin; ERV, expiratory reserve volume; F/V ex, expiratory flow/volume; FEV 1, forced expiratory volume in one second; FIF 50, 50\% of forced vital capacity; FRCpleth, functional residual capacity; FVC, forced vital capacity; Hb, haemoglobin; KCO_SB, single-breath transfer coefficient of the lung for carbon monoxide; KCOc_SB, single-breath transfer coefficient of the lung for carbon monoxide corrected for haemoglobin; LL, lower limit; MEF 50, maximal expiratory flow at 50\% of the forced vital capacity; MFEF 75/25, mid-forced expiratory flow rate of 25-75\%; PEF, peak expiratory flow; PIF, peak inspiratory flow; Pred, predicted; RV, residual volume; T, time; TLC, total lung capacity; UL, upper limit; V, volume; VA_SB, single-breath alveolar volume; $V C$, vital capacity 


\section{ANSWER 3}

A screen could include the following depending on the situation:

- autoimmune screen - antinuclear autoantibody, dsDNA, extractable nuclear antigen, rheumatoid factor, anti-cyclic citrullinated peptide

- if myositis-associated interstitial lung disease is suspected-extended myositis panel, creatine kinase

- vasculitic screen - anti-neutrophil cytoplasmic antibodies and perinuclear anti-neutrophil cytoplasmic antibodies

- if sarcoidosis is suspected - serum angiotensin-converting enzyme

- if hypersensitivity pneumonitis related to bird exposure is suspected - avian precipitins.

\section{ANSWER 4}

The clinician should consult a reliable source, such as product information or local guideline, to determine baseline evaluation and monitoring. Typically, monitoring for DIPF involves a baseline chest $\mathrm{X}$-ray and lung function test, with periodic lung function tests thereafter. Ideally the doctor initiating the medication would perform the baseline evaluation. It should be made explicit which doctor is responsible for ongoing monitoring. The patient should be educated about possible side effects and recommended monitoring. Use of a recall system would be ideal to ensure monitoring is not forgotten. A review article indicated that approximately $50 \%$ of patients beginning amiodarone received minimum baseline evaluation, and fewer than $25 \%$ received the recommended ongoing surveillance. ${ }^{3}$ In the case of long-term nitrofurantoin use, there is no Australian authority recommending routine screening with chest X-rays and/or lung function tests.

\section{ANSWER 5}

The TGA recommends a report be made for all suspected adverse events to new therapeutic goods, all suspected medicine and/or vaccine interactions, undocumented adverse events and serious adverse events. ${ }^{4}$ In this case, the adverse event was reported as the nitrofurantoin caused significant harm to the patient. Clinicians should be aware that acute, subacute and chronic pulmonary reactions have been observed with nitrofurantoin use. The estimated incidence of severe acute pulmonary reactions is estimated at one in 5000 short-term users, while chronic pulmonary reactions causing hospitalisation occur in approximately one in 750 long-term users. ${ }^{5}$

\section{Key points}

- Typical presenting symptoms of pulmonary fibrosis are dry cough and exertional dyspnoea.

- Numerous medications can cause pulmonary fibrosis, and patients require monitoring for its occurrence.

- Serious medication adverse events should be reported to the TGA.

\section{Authors}

John O'Bryen BSc, MBBS, FRACGP, FARGP, DipDerm, General Practitioner, Qld

Gwynne Hannay BEng (Medical), PhD, MBBS,

FRACGP, General Practitioner, Qld

Sarah Gleeson BMed, FRACP, Respiratory and Sleep Physician, Qld

Competing interests: None.

Funding: None.

Provenance and peer review: Not commissioned, externally peer reviewed.

Correspondence to:

j.obryen@griffith.edu.au

\section{References}

1. Skeoch S, Weatherley N, Swift AJ, et al. Druginduced interstitial lung disease: A systematic review. J Clin Med 2018;7(10):356. doi: 10.3390/ jcm7100356.

2. Camus P. Pneumotox on line: The drug induced respiratory disease website. Dijon, FR: Pneumotox 2021. Available at www.pneumotox.com [Accessed 3 April 2021].

3. Stelfox HT, Ahmed SB, Fiskio J, Bates DW. Monitoring amiodarone's toxicities: Recommendations, evidence, and clinical practice. Clin Pharmacol Ther 2004;75(1):110-22. doi: 10.1016/j.clpt.2003.09.010.

4. Therapeutic Goods Administration. Reporting adverse events involving medicines, vaccines or medical devices. Canberra, ACT: TGA, 2021 Available at www.tga.gov.au/reporting-adverseevents [Accessed 26 January 2021].

5. Jick SS, Jick H, Walker AM, Hunter JR. Hospitalizations for pulmonary reactions following nitrofurantoin use. Chest 1989;96(3):512-15. doi: 10.1378/chest.96.3.512. 